\title{
Dynamics of an enhanced network region observed in $\mathrm{H} \alpha$
}

\author{
N. $\mathrm{Al}^{1}$, C. Bendlin ${ }^{2}$, J. Hirzberger ${ }^{3}$, F. Kneer ${ }^{2}$, and J. Trujillo Bueno ${ }^{4,5}$ \\ 1 Istanbul University, University Observatory, 34452 University-Istanbul, Turkey \\ 2 Universitäts-Sternwarte, Geismarlandstr. 11, 37083 Göttingen, Germany \\ 3 Institut für Geophysik, Astrophysik und Meteorologie, Universitätsplatz 5, 8010 Graz, Austria \\ ${ }^{4}$ Instituto de Astrofísica de Canarias, 38205 La Laguna, Spain \\ 5 Consejo Superior de Investigaciones Científicas, Spain
}

Received 13 October 2002 / Accepted 13 February 2004

\begin{abstract}
This investigation is based on $\mathrm{H} \alpha$ observations of high spatial resolution. They stem from an enhanced network region near disk centre of the sun and consist of broad-band and narrow-band images taken with the two-dimensional "Göttingen" Fabry-Perot spectrometer mounted in the Vacuum Tower Telescope at the Observatorio del Teide/Tenerife. The "lambdameter method" was applied to derive intensity and velocity maps over the two-dimensional field of view reflecting the behaviour of these parameters in the solar chromosphere. The determination of the source function, the line-of-sight (LOS) velocity, the Doppler width and the optical depth was based on Beckers' cloud model (Beckers 1964). From the LOS velocity distribution along the $\mathrm{H} \alpha$ structures we conclude that ballistic motions are unlikely to prevail. Especially the bright $\mathrm{H} \alpha$ features cannot be explained by the cloud model. It is shown how, instead, two-dimensional non-LTE radiative transfer calculations of embedded chromospheric structures lead to reasonable agreement with the observed line profiles from these bright features. It is found that many of the bright fibril-like structures near dark fibrils can be explained by radiation which is blocked by absorbing material at large heights and escapes through less opaque regions. We estimate the number densities and the temperature. With these and with the measured velocities, the kinetic energy flux and the enthalpy flux related to the motions of the fine structures can be calculated. Both fall short by at least an order of magnitude of covering energy losses by radiation of active chromospheric regions.
\end{abstract}

Key words. Sun: chromosphere - Sun: faculae, plages - techniques: high angular resolution - radiative transfer

\section{Introduction}

Mottles form the most prominent structures of the solar chromosphere seen against the disk in the wings of the $\mathrm{H} \alpha$ line. They are located on the borders of supergranular cells and mostly appear in groups resembling rosettes or chains. The jet-like structures presumably extend along the magnetic field lines and cover between $7 "$ and 14". Rosettes tend to occur where three supergranulation cells meet. This location coincides with the centre of the rosettes from which mottles spread radially outwards. In the literature, a distinction between dark and bright mottles is often made and a spatial relation between them has been reported (Zachariades et al. 2001, and references therein). It is stated that they appear at the same location when projected against the solar disk but they are found in different atmospheric heights, the bright ones occurring below the dark ones. According to Tsiropoula et al. (1993), the minimum height for the dark mottles is 5000 to $7000 \mathrm{~km}$ above the upper photosphere. Another important difference between these structures lies in their line profiles. While the profiles of bright mottles exhibit an emission core and absorption in both wings,

Send offprint requests to: F. Kneer, e-mail: kneer@uni-sw.gwdg.de those of the dark ones typically show broad U-shaped profiles and lower line-centre intensities (Tsiropoula et al. 1994).

To determine the line-of-sight velocity as well as other physical quantities of chromospheric structures like the source function, the optical depth and the Doppler width, often Beckers' cloud model (1964) is employed (Grossmann-Doerth \& von Uexküll 1971, 1973; Alissandrakis et al. 1990; Tsiropoula et al. 1993, 1994; Lee et al. 2000). The most important requirements for this model are that the structures of interest are isolated and that they must be located clearly above the formation height of $\mathrm{H} \alpha$ such that they can be considered to form a cloud well separated from the lower atmospheric layers that contribute to the undisturbed $\mathrm{H} \alpha$ line. Dark mottles often fulfil this requirement and are thus appropriate candidates to enter this model - unlike the bright ones.

Mainly for diagnostics of filaments and of highly elevated filamentary structures, the original cloud model by Beckers (1964) has been refined using non-LTE radiative transfer calculations by Mein et al. (1996) who allow for a variation of the source function within the cloud. Heinzel et al. (1999) included the effect of the cloud's motion on the source function. A grid of cloud-like models using non-LTE multi-level radiative 


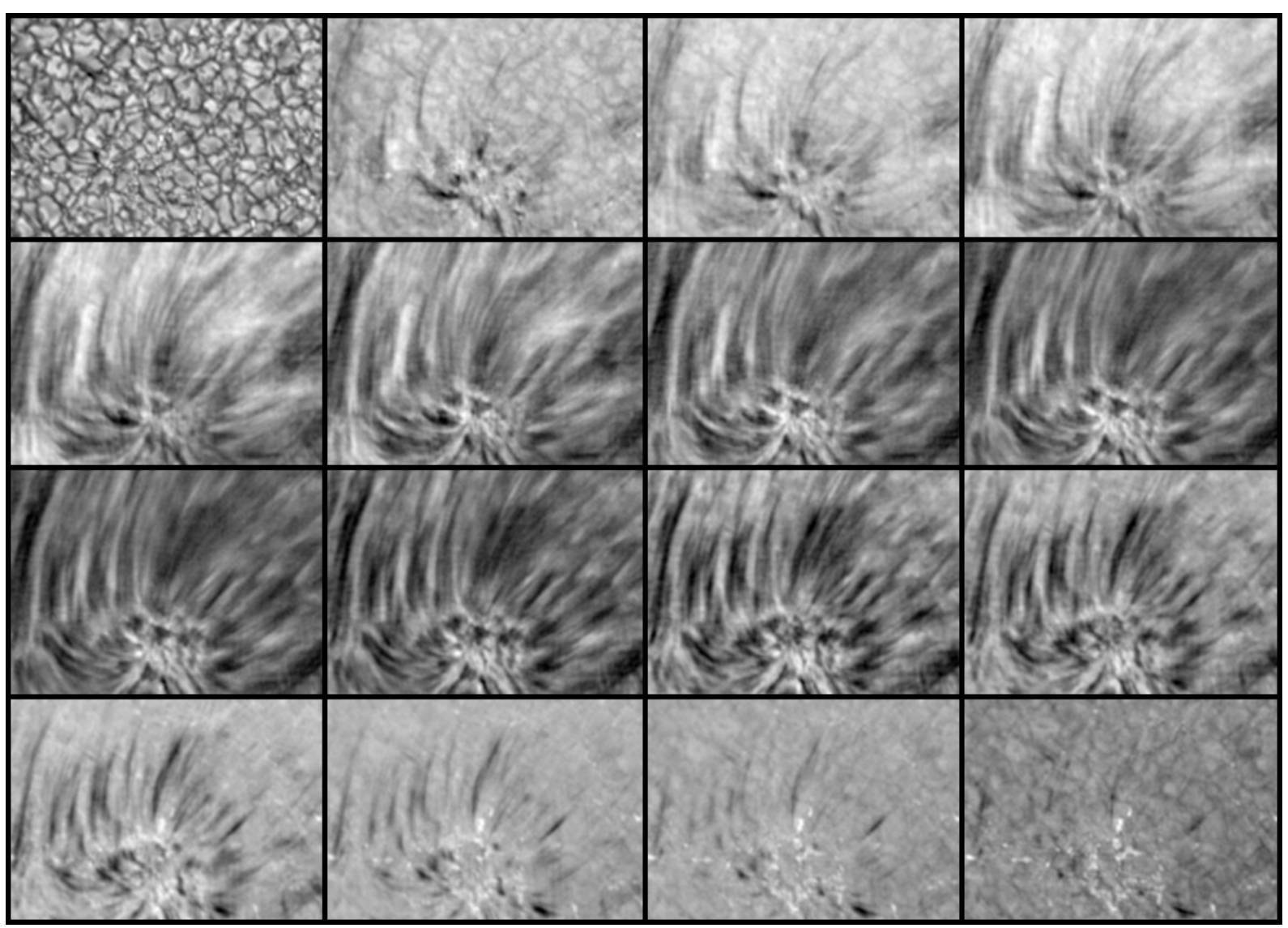

Fig. 1. Reconstructed white-light image (upper left) and narrow-band scan belonging to the same field of view. The size of each image is 32 .' $2 \times 23^{\prime \prime}$. 0 . The wavelength positions of the narrow-band filtergrams decrease image by image by $125 \mathrm{~m} \AA$ with the last image in the second row corresponding closest to the centre of the mean line profile.

transfer was calculated by Molowny-Horas et al. (1999) and was applied by these authors and by Schmieder et al. (2003) for inversion of filament data.

Cram (1975) questioned the cloud model by demonstrating that line profiles interpreted by high lying clouds could as well originate from low structures embedded in the atmosphere from which the undisturbed $\mathrm{H} \alpha$ profile originates. We shall comment on this criticism below when we describe our two-dimensional (2D) non-LTE calculations.

This investigation deals with the application of the cloud model to highly resolved $\mathrm{H} \alpha$ data and the resulting variation of the quantities mentioned above within the structures under investigation, i.e. dark mottles. We will restrict ourselves to constant source functions along the line of sight within the dark mottles as in the original cloud model. But in addition, we shall show by means of 2D non-LTE radiative transfer calculations how bright $\mathrm{H} \alpha$ structures can be reproduced by parameter variations within the atmosphere forming the undisturbed $\mathrm{H} \alpha$ profile. In Sect. 2, we report on the observation and data reduction. Section 3 deals with the analysis and results obtained in various ways: by the "lambdameter" method (Sect. 3.1), by the cloud model in terms of atmospheric parameters in elevated structures (Sect. 3.2) and with 2D non-LTE simulations and the interpretation of bright structures (Sect. 3.3). Section 4 concludes the paper.

\section{Observation and data reduction}

We refer to $\mathrm{Al}$ et al. (2003) for a more detailed description of the observations. They were taken in May 2002 from an enhanced network region near disk centre in $\mathrm{H} \alpha$ with the twodimensional "Göttingen" spectrometer mounted in the Vacuum Tower Telescope (VTT) at the Observatorio del Teide on Tenerife/Spain (Bendlin et al. 1992; Bendlin \& Volkmer 1995; Koschinsky et al. 2001). The data consist of scans of short exposure, narrow-band $(\Delta \lambda=72 \mathrm{~m} \AA)$ images at 18 equidistant $(\Delta \lambda=125 \mathrm{~m} \AA)$ positions in $\mathrm{H} \alpha$ and simultaneous broad-band images. The necessary dark and flat field frames were taken as well.

After correction for dark signal, flat fields and image motion, the white-light images were restored using the "spectral ratio" (von der Lühe 1984) and the "speckle masking" methods (Weigelt 1977). The rather complicated reduction procedure required for the narrow-band images has been described in detail by Hirzberger et al. (2001). The optical transfer function derived from the restoration of the white-light images was finally applied to reconstruct the narrow-band images (cf. also $\mathrm{Al}$ et al. 2003). The narrow-band images were filtered to exhibit structures of a size $\geq 0$ '. 4 . A final field of view $(F O V)$ of 32 .' $2 \times 23^{\prime \prime}$. 0 remained for further analysis.

Figure 1 displays the reconstructed images through one scan as described above. The first panel represents the 

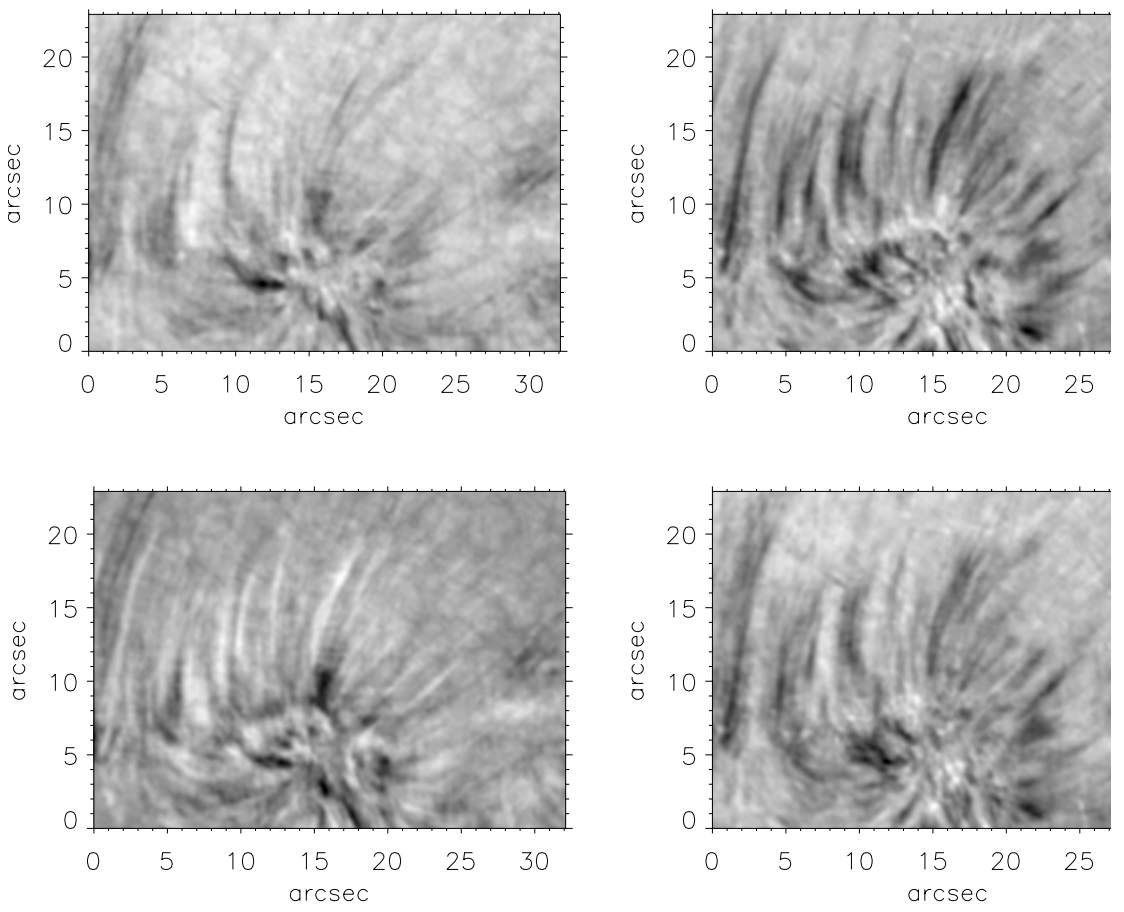

Fig. 2. Intensity images at $\mathrm{H} \alpha+0.6 \AA$ and $\mathrm{H} \alpha-$ $0.6 \AA$ (upper left and right panels) and resulting Doppler and intensity image (lower left and right panels) obtained by subtraction and addition of the upper two images, respectively.

reconstructed white-light image followed by the narrow-band images belonging to the same field of view. The wavelength decreases row by row from the left to the right. The last image in the second row corresponds best to the spectral position of the centre of the mean line profile. The lower part of the images taken in both wings exhibits a conspicuous roundish bright area from which longish structures extend radially outwards, giving the appearance of enhanced network. Here, a rosette has formed. Presumably, this is a location where three supergranulation cells have met. At the centre of the rosette, the reconstructed broad-band image shows filigree which can also be seen in some narrow-band images, preferably in the blue wing.

From the intensity values of the narrow-band images belonging to different wavelength positions, a line profile was reconstructed for each pixel. Intensities in between the 18 wavelength positions were obtained by spline interpolation. The so-called "lambdameter method" (Tsiropoula et al. 1993) was used to derive two-dimensional intensity and velocity maps at intensity levels where the (full) profile widths are $0.51 \AA$, $0.765 \AA, 1.020 \AA$, and $1.275 \AA$. The calculation of the line-ofsight velocity is based on an averaged reference profile taken from the upper right corner of the $F O V$ showing no significant structure.

\section{Results}

\subsection{Lambdameter method and Doppler images}

Figure 2 exhibits the intensity images for $\mathrm{H} \alpha+0.6 \AA$ and $\mathrm{H} \alpha-0.6 \AA$ as well as the Doppler image, obtained by subtraction $((\mathrm{H} \alpha+0.6 \AA)-(\mathrm{H} \alpha-0.6 \AA))$, and the intensity image, representing the sum of the images $((\mathrm{H} \alpha+0.6 \AA)+(\mathrm{H} \alpha-0.6 \AA))$. In the Doppler image, darker and lighter shades of grey indicate downflows and upflows, respectively. The arch-shaped, radially extended structures with a width of about 0.5 to 1. .'0 and

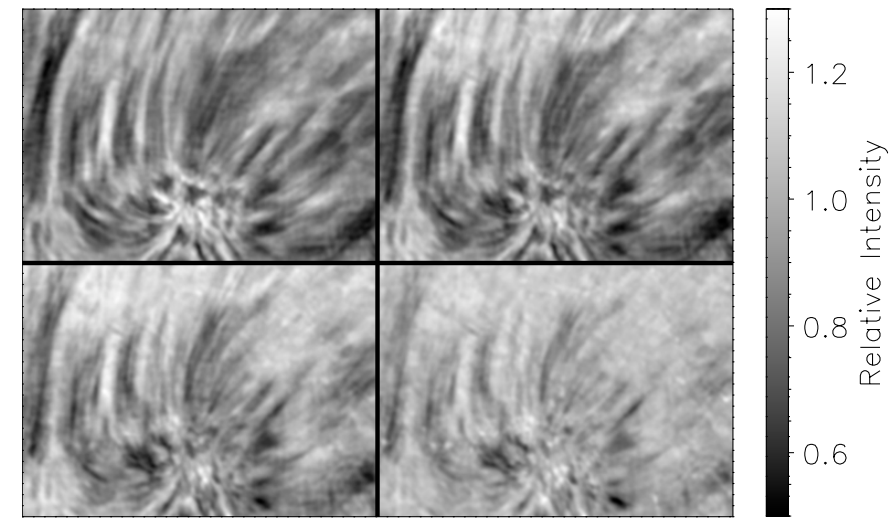

Fig. 3. Intensity images belonging to the profile widths of $0.510 \AA$, $0.765 \AA, 1.020 \AA$, and $1.275 \AA$ (upper left and right and lower left and right panel, respectively).

a length of $5^{\prime \prime}$ to $10^{\prime \prime}$ in the lower part of the FOV therefore exhibit upflows.

While Fig. 3 shows the intensity images taken from the profile widths of $0.510 \AA, 0.765 \AA, 1.020 \AA$, and $1.275 \AA$, Fig. 4 represents the corresponding velocity images obtained by the lambdameter method described by Tsiropoula et al. (1993). It relates the wavelength shifts of the bisectors at the profile widths given above with respect to the average profile (from an undisturbed area) to Doppler shifts. The upper left and lower right image thus correspond to the highest and lowest atmospheric layer, respectively. Both the intensity and the Doppler images outline well the filamentary $\mathrm{H} \alpha$ structures surrounding the centre of the rosette. As will be argued in the discussion of the "cloud model" below, the reader must be cautioned against a too literal interpretation of Doppler images, shown in Fig. 4, in terms of velocity values. With this reservation, we give the extreme values. The maximum and minimum 


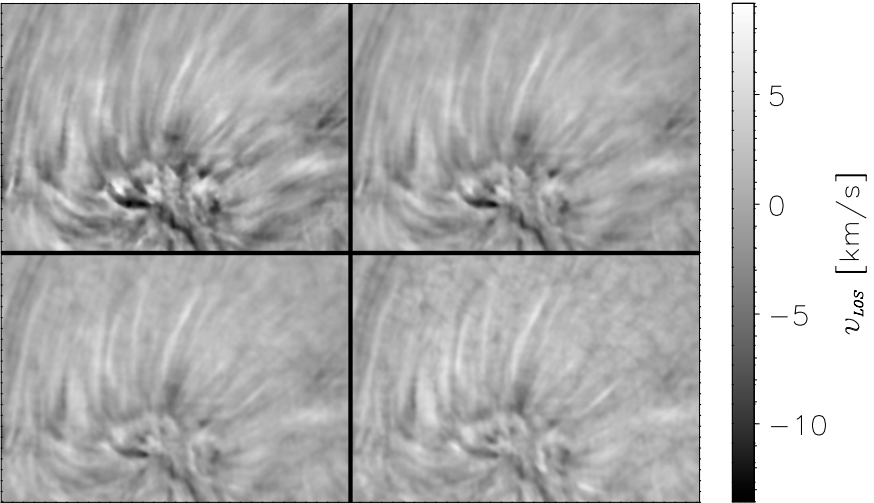

Fig. 4. Line-of-sight velocity $v_{\text {LOS }}$ obtained by the lambdameter method for the same spectral positions and in the same order as in Fig. 3.

velocities in these four images are 9.2 and $-13.5 \mathrm{~km} \mathrm{~s}^{-1}$, 6.3 and $-12.5 \mathrm{~km} \mathrm{~s}^{-1}, 6.0$ and $-9.8 \mathrm{~km} \mathrm{~s}^{-1}$, and 7.0 and $-7.0 \mathrm{~km} \mathrm{~s}^{-1}$ in the given order. Figure 4 indicates that the downflow occurring in the central part of the rosette is more pronounced in higher than in lower layers. The mean velocity of the arch-shaped structures best seen in the lower two velocity images (and possibly representing dark mottles) reaches about $5 \mathrm{~km} \mathrm{~s}^{-1}$.

\subsection{Cloud model}

The "cloud model" was applied to check the velocity values and to derive several other physical quantities helpful for the investigation of the solar chromosphere. The model was employed within the limits discussed by Alissandrakis et al. (1990). Accordingly, when calculating the intensity $I(\lambda)$ emerging from a cloud with source function $S$ irradiated from below by the reference intensity $I_{0}(\lambda)$, the contrast profile $C(\lambda)$ is given by:

$C(\lambda)=\frac{I(\lambda)-I_{0}(\lambda)}{I_{0}(\lambda)}=\left(\frac{S}{I_{0}(\lambda)}-1\right) \cdot\left(1-\mathrm{e}^{-\tau(\lambda)}\right)$,

where

$\tau(\lambda)=\tau_{0} \cdot \exp \left[-\left(\frac{\lambda-\lambda_{\mathrm{c}}\left(1-v_{\mathrm{LOS}} / c\right)}{\Delta \lambda_{\mathrm{D}}}\right)^{2}\right]$

describes the wavelength dependence of the optical thickness $\tau$ by a Gaussian. Here, $\tau_{0}$ stands for the optical depth at the line centre, $\lambda_{\mathrm{c}}$ for the wavelength of the line centre, $\lambda_{\mathrm{c}}\left(v_{\mathrm{LOS}} / c\right)$ for the shift due to the macroscopic LOS velocity $v_{\mathrm{LOS}}$, and $\Delta \lambda_{\mathrm{D}}$ for the Doppler width. (Our convention is that velocities are positive towards the observer.) The undisturbed reference profile $I_{0}(\lambda)$ is taken from the upper right corner in the $F O V$. The model is based on the assumption that the velocity, the Doppler width and the source function are constant within the cloud along the line of sight (as a zero-order approximation).

Figure 5 depicts typical contrast profiles observed in dark mottles. The accuracy of the contrast measurements is 0.02 . Two conditions had to be fulfilled by pixels in the $F O V$ for the cloud model to be applied: firstly, the structures had to be

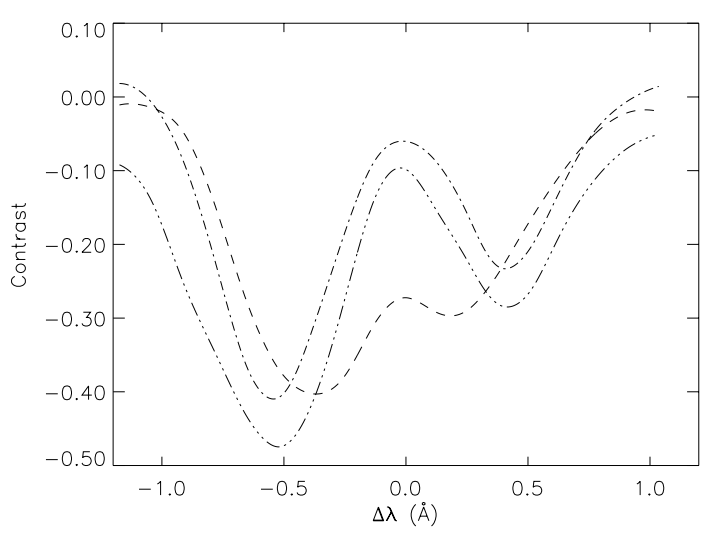

Fig. 5. Typical contrast profiles observed in dark mottles. $\Delta \lambda=\lambda-\lambda_{\mathrm{c}}$ is the wavelength distance from the line centre of the reference profile.

dark in the intensity image of $\mathrm{H} \alpha \pm 0.6 \AA$ of Fig. 2, lower right panel, i.e. we considered pixels with an intensity of less than 0.9 of the average intensity $I_{0}(\mathrm{H} \alpha \pm 0.6 \AA)$ to be dark. Secondly, we required that the contrast $C(\lambda)<0.1$. The parameters were determined by an iterative least squares fitting procedure. After the calculation, they were checked according to the criteria given by Alissandrakis et al. (1990) and Tsiropoula et al. (1994). Only pixels with a corresponding source function $S<0.2$ (in units of the continuum intensity) and an optical depth $\tau_{0}<5$ were selected for further analysis. The number of pixels fulfilling all requirements was 25190 out of 74060 .

Figure 6 shows, from top to bottom, the histograms of the cloud velocity, the source function, the optical depth, and the Doppler width. Most clouds have LOS velocities near $0 \mathrm{~km} \mathrm{~s}^{-1}$. The whole range of velocities includes -22 to $+21 \mathrm{~km} \mathrm{~s}^{-1}$. The distribution for the source function reaches its peak within the range of $0.13-0.15$ (in units of the continuum intensity $I_{\mathrm{c}}$ ), for the optical depth within 1.3-1.7, and for the Doppler width in the range of $0.38-0.43 \AA$.

The mean values for the distributions in Fig. 6 are $0.18 \mathrm{~km} \mathrm{~s}^{-1}, 0.14 I_{\mathrm{c}}, 1.58$, and $0.44 \AA$, while Tsiropoula et al. (1993) gave $-0.26 \mathrm{~km} \mathrm{~s}^{-1}, 0.163 I_{\mathrm{c}}, 1.8$, and $0.37 \AA$ for dark mottles. Lee et al. (2000) also investigated dark mottles and found a mean value for the optical depth of 2.2. In their data, the velocities varied between -15 and $+10 \mathrm{~km} \mathrm{~s}^{-1}$ and the peak of the velocity distribution appeared at $-1.9 \mathrm{~km} \mathrm{~s}^{-1}$. For the calculation of the energy transported in the mottles, we will use below the average modulus of the velocities, for which we find $\overline{\left|v_{\mathrm{LOS}}\right|}=3.96 \mathrm{~km} \mathrm{~s}^{-1}$. We note that the actual velocities along the $\mathrm{H} \alpha$ fibrils may be substantially different from LOS velocities, i.e. they may be much larger than $\overline{\left|v_{\mathrm{LOS}}\right|}$ and we may expect values of $20 \mathrm{~km} \mathrm{~s}^{-1}$ when observing under different viewing angles. Sometimes, such high velocities do indeed occur in the cloud model fits and are also estimated for spicules (e.g. Beckers 1972).

Figure 7 depicts contours of the LOS velocity resulting from the the cloud model. The highest velocities do not necessarily occur at the ends of the fibrils, which one may regard as their footpoints. With the picture in mind that the mottles exhibit elevated arched structures above the atmosphere, the high velocities at the top of the arches would not allow an 

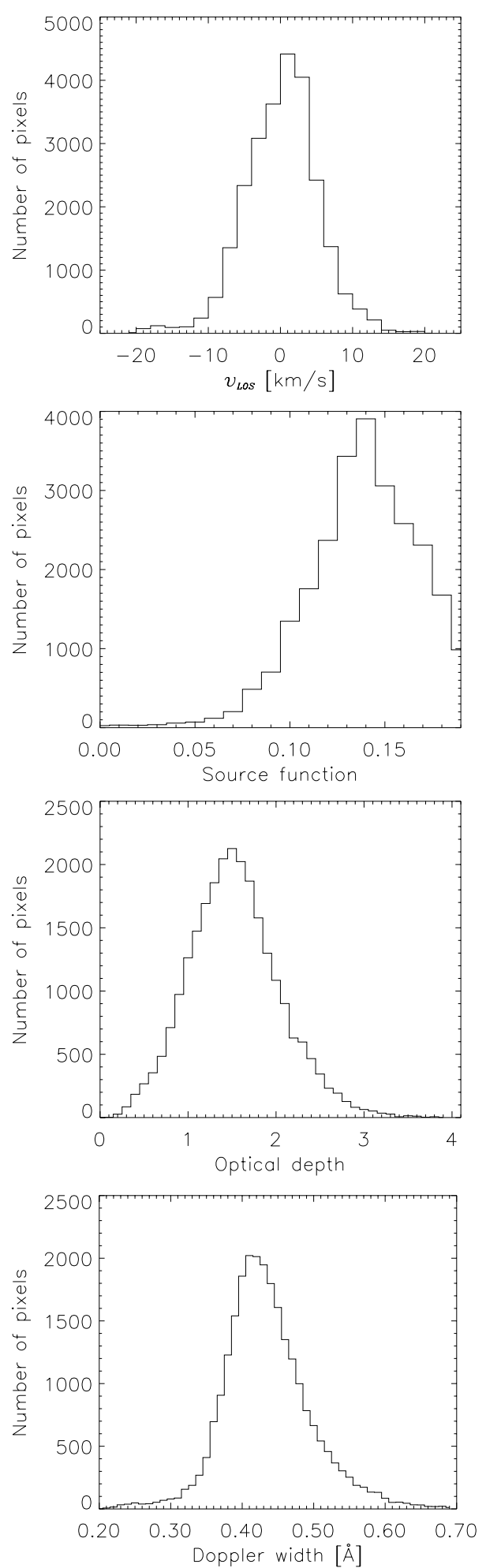

Fig. 6. Histograms of line-of-sight velocity $v_{\mathrm{LOS}}$, source function $S$ in units of the continuum intensity $I_{\mathrm{c}}$, optical depth $\tau_{0}$, and Doppler width $\Delta \lambda_{\mathrm{D}}$

interpretation in terms of ballistic motions as suggested by Christopolou et al. (2001) from measurements of spicules.

The mean values of the optical depth (1.58) and of the Doppler width $(0.44 \AA)$ were used to derive the product of the number density of the second level in hydrogen $N_{2}$ and

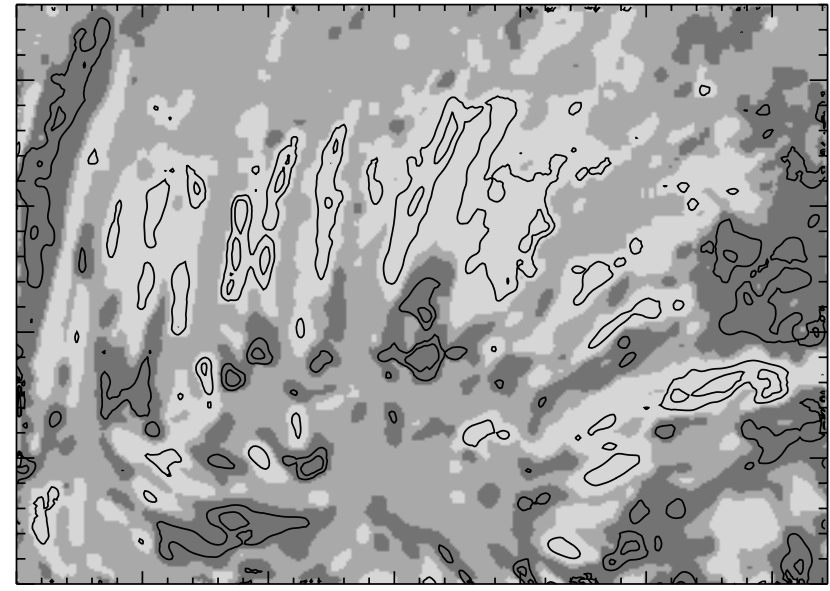

Fig. 7. Contour plot of the line-of-sight velocity $v_{\text {LOS }}$. The bright and dark patches indicate upflows and downflows with velocities $\geq+1$ and $\leq-1 \mathrm{~km} \mathrm{~s}^{-1}$, respectively. They are overlaid with contours of \pm 5 and $\pm 10 \mathrm{~km} \mathrm{~s}^{-1}$. In the medium grey areas either the velocities lie between +1 and $-1 \mathrm{~km} \mathrm{~s}^{-1}$ or the cloud model fit was not applied at all. The tickmarks are separated by $1^{\prime \prime}$.

the geometrical width $L$ of the structures under investigation. Neglecting stimulated emission, the optical depth is given by

$\tau(\lambda)=\left(\frac{\pi e^{2}}{m_{\mathrm{e}} c^{2}}\right) f \lambda^{2} \phi(\lambda) N_{2} L$,

where the absorption profile $\phi(\lambda)$ is normalized such that, with the Gaussian according to Eq. (2),

$\int \phi(\lambda) \mathrm{d} \lambda=1$ and $\phi(0)=\frac{1}{\sqrt{\pi} \Delta \lambda_{\mathrm{D}}}$.

Inserting the constants in Eq. (3), for which we used the common symbols, one obtains

$\tau_{0}=1.38 \times 10^{-21} \frac{N_{2} L}{\Delta \lambda_{\mathrm{D}}}$.

With the average values of $\tau_{0}$ and $\Delta \lambda_{\mathrm{D}}$ given above we obtain $N_{2} L \approx 5.1 \times 10^{12} \mathrm{~cm}^{-2}$.

For $L$, a width of $700 \mathrm{~km}$ may be assumed ( $\left(1^{\prime \prime}\right.$, cf. Fig. 2$)$, leading to a density $N_{2} \approx 7 \times 10^{4} \mathrm{~cm}^{-3}$. For the purpose of estimating energy fluxes transported by the moving fine structures we need their temperature and total particle density. We adopt an approximate chromospheric temperature of $7000 \mathrm{~K}$ from the VALC model of Vernazza et al. (1981). Furthermore, we assume that the excitation of the second level of hydrogen, i.e. the ratio $N_{2} / N_{1}$, does not depend much on the assumption of hydrostatic equilibrium. From the same VALC model and for this specific density of $N_{2}$, we then get a total hydrogen density of $N_{\mathrm{H}} \approx 4.5 \times 10^{11} \mathrm{~cm}^{-3}$ and an electron density $N_{\mathrm{e}} \approx 5 \times 10^{10} \mathrm{~cm}^{-3}$.

Since the VALC model describes the average, homogeneous chromosphere, a comparison of the temperature and the electron density obtained from it with values found in spicules is appropriate. Beckers (1972) gives for the latter temperatures in the range of $9000-16000 \mathrm{~K}$ while the electron density in spicules varies between $1.6 \times 10^{11} \mathrm{~cm}^{-3}$ and $3.4 \times 10^{10} \mathrm{~cm}^{-3}$. 
Matsuno \& Hirayama (1988) give temperatures in spicules of $5200-9000 \mathrm{~K}$. From the high electron density in spicules and with an ionization degree of 0.88 (Beckers 1972), one obtains a total hydrogen density of $N_{\mathrm{H}} \approx 1.8 \times 10^{11} \mathrm{~cm}^{-3}$, i.e. a value somewhat lower than the one from the VALC model. The energy fluxes with densities from the latter model will thus represent upper limits.

Furthermore, we estimate the kinetic energy flux and the enthalpy flux in the moving mottles. The needs to cover the chromospheric radiative losses of enhanced network (or plages) are approximately a factor of 10 larger than that of quiet chromospheric regions and thus amount to

$F_{\text {rad }} \approx 5 \times 10^{7} \mathrm{erg} \mathrm{cm}^{-2} \mathrm{~s}^{-1}$

(see e.g., Athay 1976; Orrall \& Kopp 1977; Fawzy et al. 2002). Taking the mean velocity of the clouds $\overline{\left|v_{\text {LOS }}\right|}$ from above at face value and using the estimated densities and temperature we obtain a kinetic energy flux of

$F_{\text {kin }}=\frac{\rho}{2} \bar{v}^{3} \approx 3 \times 10^{4} \mathrm{erg} \mathrm{cm}^{-2} \mathrm{~s}^{-1}$.

For the enthalpy flux one finds

$F_{\text {ent }}=\frac{5}{2} N_{\text {tot }} k T \bar{v} \approx 5 \times 10^{5} \mathrm{erg} \mathrm{cm}^{-2} \mathrm{~s}^{-1}$.

Both values fall short of the needs to cover the radiative losses. When adopting a much higher average velocity of $20 \mathrm{~km} \mathrm{~s}^{-1}$ we get $F_{\text {kin }} \approx 3.8 \times 10^{6} \mathrm{erg} \mathrm{cm}^{-2} \mathrm{~s}^{-1}$ and $F_{\text {ent }} \approx$ $2.5 \times 10^{6} \mathrm{erg} \mathrm{cm}^{-2} \mathrm{~s}^{-1}$. Hence, neither the kinetic energy nor the enthalpy can balance the chromospheric radiative losses. Although von Uexküll et al. (1989) estimated the kinetic energy flux an order of magnitude lower than the one given above in Eq. (7), they pointed out that the magnetic energy supply indicated by the motions could still be sufficient.

\subsection{Embedded structures}

Many contrast profiles could not be fitted by the cloud model. These obviously stem from bright structures with high positive contrast either near line centre of $\mathrm{H} \alpha$ or in the wings. Some examples are given in Fig. 8 together with two-dimensional (2D) non-LTE calculations of contrast profiles from embedded structures which we shall discuss now.

We use the equivalent two-level atomic model for the $\mathrm{H} \alpha$ line source function $S_{1}$ (cf. Mihalas 1970)

$S_{1}=\frac{\int J_{\lambda} \phi(\lambda) \mathrm{d} \lambda+\varepsilon B+\eta B^{*}}{1+\varepsilon+\eta}$,

where $\eta$ and $B^{*}$ contain the radiative and collisional routes to and from the continuum level of hydrogen to the second and third level. The other terms in Eq. (9) have their usual meanings. Continuous background absorption and emission in LTE are added to get the wavelength-dependent source function

$S_{\lambda}=\kappa_{\lambda} S_{1} /\left(\kappa_{\lambda}+\kappa_{\mathrm{c}}\right)+\kappa_{\mathrm{c}} B /\left(\kappa_{\lambda}+\kappa_{\mathrm{c}}\right)$,

where $\kappa_{\lambda}$ and $\kappa_{\mathrm{c}}$ stand for the line absorption and continuous absorption coefficient, respectively.
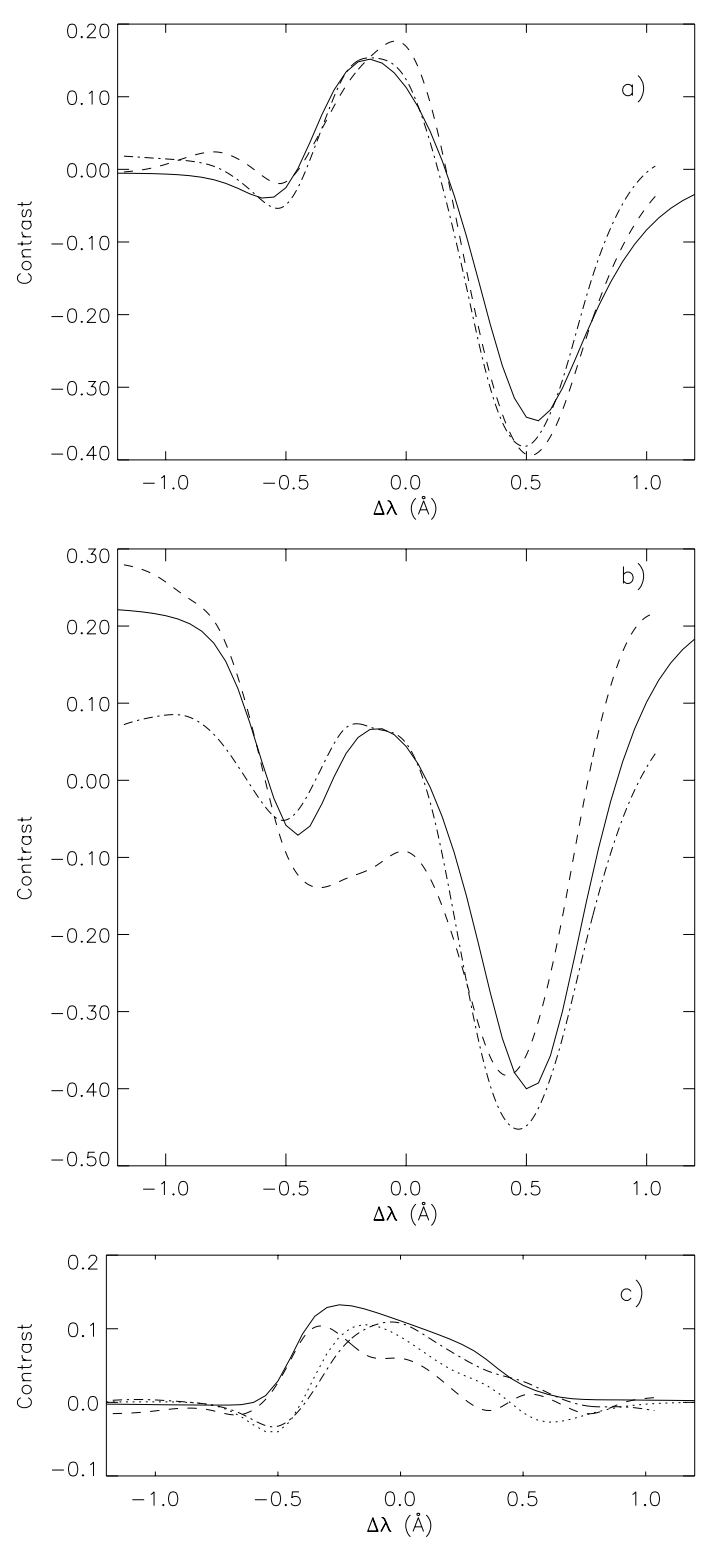

Fig. 8. Contrast profiles not explicable by the cloud model; dashed and dash-dotted lines: measured contrasts; solid and dotted lines: profiles from 2D non-LTE calculations with parameters described in the text.

The standard values, i.e. from the unperturbed atmosphere, of $\varepsilon, B, \eta$, and $B^{*}$ are calculated from the VALC model (Vernazza et al. 1981) with radiation temperatures of $5800 \mathrm{~K}$ and $5600 \mathrm{~K}$ in the Balmer and Paschen continua, respectively. The damping constant and the Doppler width for the absorption profiles (we used the Voigt function) were adjusted to give a good fit of the line profile calculated with the VAL C model to the profile from the Fourier Transform Spectrometer Atlas by Brault \& Neckel (1987).

Throughout the chromosphere, i.e. at heights between 500 and $2100 \mathrm{~km}$ above $\tau_{5000}=1$ in the VAL C atmospheric model, the population routes to and from the hydrogen continuum level dominate the source function. $\eta$ is almost constant, a factor of 2.5 larger than $\varepsilon$ (the collisional route between hydrogen levels two and three) at $500 \mathrm{~km}$ and a factor of 10 larger at the top of the atmosphere. The Planck function $B$ and the ionization 
radiation field $B^{*}$ do not differ much where the latter is also almost constant throughout the chromosphere. In the deep photosphere, LTE prevails and the source function $S$ approaches the Planck function. It is thus difficult to conceive a mechanism which increases the $\mathrm{H} \alpha$ source function in the chromosphere as suggested by Cram (1975) in his models.

The relevant atmospheric and line parameters (line and continuous opacities, damping constant, Doppler width, $\varepsilon, \eta, B$, $\left.B^{*}\right)$ were transferred to a $2 \mathrm{D}$ periodic model with a horizontal period of $X=1800 \mathrm{~km}$. For the recalculation of the source function, these parameters (except the macroscopic LOS velocity, see below) were then changed by fluctuations of the form

$\delta P(z, x) / P(z)=f(z) \times \exp \left[-((x-X / 2) / \Delta x)^{2}\right]$,

where $P$ denotes the parameter under consideration, $f(z)$ the vertical functional form of the fluctuations, and $\Delta x$ its horizontal extent. The fluctuations are thus represented by their positions and widths in $z$ and $x$ and by their amplitude.

The source function according to Eq. (10) was calculated with the 2D non-LTE code developed by Auer et al. (1994). It uses periodic boundary conditions and finds the self-consistent solution iteratively. With Ng's (1974) acceleration technique, convergence to a maximum relative change of some $10^{-5}$ is achieved within 15 iterations and within $20 \mathrm{~s}$ on a fast PC for the present problem.

Once the source function is known everywhere, we calculate the emergent line profiles along the $z$ axis at various $x$ positions. Here, LOS velocities were introduced as Doppler shifts in the absorption profiles. According to the "dynamic models" by Heinzel et al. (1999), this is not fully consistent since Doppler shifts of the line absorption influence the source function. However, in all one-dimensional test cases studied, such a change did not alter the resulting contrast profiles beyond the accuracy of the measurements.

Three of the simulated contrast profiles $C(\lambda)$ in Fig. 8 were calculated in the middle of the periodic fluctuations using the emerging intensity $I_{0}(\lambda)$ from the unperturbed $2 \mathrm{D}$ atmospheric model. These are the solid profiles in Figs. $8 \mathrm{a}$ and $8 \mathrm{~b}$ and the dotted profile in Fig. 8c. Note that the solid profile in Fig. 8c stems from the edge of the $x$ range, i.e. from $x=0$. We consider the simulations as a demonstration of dynamic inhomogeneities embedded in the otherwise unperturbed atmosphere. Throughout the following discussion of the results shown in Figs. $8 \mathrm{a}-\mathrm{c}$ it should be kept in mind that it has not been our intention to fit exactly the measurements. We rather wished to explore the effects of various parameters by introducing variations compatible with what is already known about magnetic flux tubes.

\subsubsection{High central emission}

Figure 8a shows two observed, asymmetric contrast profiles with high central emission. The simulation (solid line in Fig. 8a) is characterized by

$$
\begin{aligned}
\delta\left(\Delta \lambda_{\mathrm{D}}(z, x)\right) / \Delta \lambda_{\mathrm{D}}(z)= & 0.4 \times \exp [-(2100 \mathrm{~km}-z) / 700 \mathrm{~km}] \\
& \times \exp \left[-((x-X / 2) / 350 \mathrm{~km})^{2}\right],
\end{aligned}
$$

$\delta\left(\kappa_{\lambda}(z, x)\right) / \kappa_{\lambda}(z)=0.8 \times \exp [-(2100 \mathrm{~km}-z) / 700 \mathrm{~km}]$

$$
\times \exp \left[-((x-X / 2) / 350 \mathrm{~km})^{2}\right] \text {, }
$$

and

$v_{\mathrm{LOS}}(z, x)=-25 \mathrm{~km} \mathrm{~s}^{-1} \times \exp [-(2100 \mathrm{~km}-z) / 250 \mathrm{~km}]$.

The embedded structures have a horizontal FWHM of approximately $580 \mathrm{~km}$. The parameters imply injection from above into the atmosphere of highly turbulent $\left(\delta\left(\Delta \lambda_{\mathrm{D}}\right) / \Delta \lambda_{\mathrm{D}}(z)=0.4\right.$, possibly hot $)$ and dense $\left(\delta \kappa_{\lambda}(z) / \kappa_{\lambda}(z)=0.8\right)$ material with high speed $\left(v_{\mathrm{LOS}}=-25 \mathrm{~km} \mathrm{~s}^{-1}\right)$. The high central emission is produced by the increased Doppler width connected with the structure, the absorption in the wings by the additional absorbing material with its high Doppler width, and the strong asymmetry by the flow.

\subsubsection{Moustache profiles}

The contrast profiles in Fig. $8 \mathrm{~b}$ are typical for the moustache phenomenon with high intensities in the damping wings (cf. Martres \& Bruzek 1977; Denker et al. 1995, and references therein). The brightening of small-scale structures, especially in the blue wing, can be seen in Fig. 1.

To produce the high intensity wings we decrease the opacity $\kappa=\kappa_{1}+\kappa_{\mathrm{c}}$ at low heights by

$$
\begin{aligned}
\delta(\kappa(z, x)) / \kappa(z)=- & 0.5 \times \exp \left[-((z-0 \mathrm{~km}) / 500 \mathrm{~km})^{2}\right] \\
& \times \exp \left[-((x-X / 2) / 250 \mathrm{~km})^{2}\right],
\end{aligned}
$$

which has a $F W H M$ in the $x$ direction of $410 \mathrm{~km}$. At the same time, we increase the Planck function in the photosphere, where the wings are formed nearly in LTE, by:

$$
\begin{aligned}
& \delta(B(z, x)) / B(z)=0.25 \times \exp \left[-((z-300 \mathrm{~km}) / 250 \mathrm{~km})^{2}\right] \\
& \times \exp \left[-((x-X / 2) / 250 \mathrm{~km})^{2}\right] .
\end{aligned}
$$

To achieve large asymmetric absorption near line centre, we introduce a strongly absorbing, downflowing inhomogeneity at the top of the atmosphere with the parameters

$$
\begin{aligned}
\delta\left(\Delta \lambda_{\mathrm{D}}(z, x)\right) / \Delta \lambda_{\mathrm{D}}(z)= & 0.4 \times \exp [-(2100 \mathrm{~km}-z) / 800 \mathrm{~km}] \\
& \times \exp \left[-((x-X / 2) / 250 \mathrm{~km})^{2}\right]
\end{aligned}
$$

$$
\begin{aligned}
\delta\left(\kappa_{\lambda}(z, x)\right) / \kappa_{\lambda}(z)=2.5 & \times \exp [-(2100 \mathrm{~km}-z) / 800 \mathrm{~km}] \\
& \times \exp \left[-((x-X / 2) / 350 \mathrm{~km})^{2}\right],
\end{aligned}
$$

and

$v_{\mathrm{LOS}}(z, x)=-18 \mathrm{~km} \mathrm{~s}^{-1} \times \exp [-(2100 \mathrm{~km}-z) / 250 \mathrm{~km}]$.

We note that, in the framework of our $\mathrm{H} \alpha$ non-LTE calculations, the reduction of the opacity and the increase of the Planck function, i.e. temperature, at low atmospheric heights are the only ways to enhance the wing intensities. We thus suggest that these moustache structures mark the location of small-scale magnetic flux tubes. The reduced line and continuum opacities $\delta\left(\kappa_{1}+\kappa_{\mathrm{c}}\right)$ by a factor of two mimic the reduced gas pressure inside the flux tube such that magnetic and internal gas pressure balance the outer gas pressure at low heights. As demonstrated 
by, e.g., Bellot Rubio et al. (1997), such magnetic structures exhibit enhanced temperature in some parts of the photosphere. From 2D radiative flux calculations, Fabiani Bendicho et al. (1992) inferred heating of the middle photospheric layers by the radiation from a partially evacuated flux tube, or flux sheet in their case. The reduced opacity shifts the height of formation to deep layers with higher temperatures and thus results in increased intensity. The enhanced Planck function in the middle photosphere as in Eq. (16) increases the intensity by approximately the same amount.

\subsubsection{Pure central emission}

Finally, the contrast profiles in Fig. 8c show almost pure emission, albeit asymmetric, near line centre. We found parameters for their simulation with the physical picture of injection of hot material from the transition region. The gas should have less line opacity and an increased background radiation field in the Balmer continuum for which one might adopt intense irradiation in the UV. This could arise from a process like an explosive event (Dere et al. 1989) interpreted by Innes et al. (1997) as magnetic reconnection. This scenario is reflected by the dotted contrast profile in Fig. 8c.

However, there is a much simpler, hence more appealing explanation. The highly absorbing feature at large heights introduced in the previous Sect. 3.3.2 to explain the low intensity at the line centre of moustache profiles impedes the radiation to escape directly to the surface. Instead, Cannon's (1970) effect of channelling of photons into less opaque regions produces increased intensity in the neighbourhood of the absorbing feature. The result is seen in the solid contrast curve in Fig. 8c. It stems from the same simulation as the one shown in Fig. $8 \mathrm{~b}$ but from the boundary of the $x$ range, away from the perturbed part of the atmosphere. To achieve the asymmetry, a moderate velocity field was introduced in the calculation given by:

$$
\begin{aligned}
v_{\mathrm{LOS}}(z, x)= & \left.-3 \mathrm{~km} \mathrm{~s}^{-1} \times \exp [-(2100 \mathrm{~km}-z) / 250 \mathrm{~km})\right] \\
& +2 \mathrm{~km} \mathrm{~s}^{-1} \times \exp \left[-((z-500 \mathrm{~km}) / 350 \mathrm{~km})^{2}\right]
\end{aligned}
$$

which represents some downflow at the top of the atmosphere and an upflow in the upper photosphere/lower chromosphere.

We propose that many of the bright fibrils next to the dark fine mottles especially well seen near line centre in Fig. 1, are produced by this non-LTE, $2 \mathrm{D}$ radiative transfer behaviour, by Cannon's (1970) channelling effect.

We estimated the energy fluxes in the embedded structures and come to the same conclusion as for the cloud model analysis above. Neither the kinetic energy flux nor the enthalpy flux carried by the structures are sufficient to cover the radiative energy flux.

\section{Conclusions}

By means of two-dimensional spectroscopy and image reconstruction with speckle methods, we obtained $\mathrm{H} \alpha$ data from an enhanced network region in the solar chromosphere of outstanding spatial resolution. This area shows abnormal granulation, many moustaches (or Ellerman bombs), and long $\mathrm{H} \alpha$ fibrils. The $\mathrm{H} \alpha$ profiles from each pixel in the field of view were subject to several models which led to the following results and conclusions:

1. The "lambdameter" method gave views of the intensities and of the line shifts at several positions in the line profile. They indicate that downflows towards the centre of the network rosette are higher at high chromospheric layers than at low layers.

2. The dark $\mathrm{H} \alpha$ mottles were analysed by fits of the contrast profiles with Beckers' (1964) cloud model in its standard form. The LOS velocities are not only high at the ends of the fibrils, but also in their middle parts. This excludes ballistic motions. Estimates of the kinetic energy and enthalpy fluxes show that these cannot balance the radiative energy losses of the (active) chromosphere.

3. Many contrast profiles could not be explained by the cloud model. We thus simulated typical examples via non-LTE, two-dimensional (2D) radiative transfer by inhomogeneities embedded in the unperturbed atmosphere. Without aiming at close fits to the observed profiles, we demonstrated the viability of such a procedure with reasonable atmospheric and dynamic parameters.

To produce high positive contrasts near the line centre of $\mathrm{H} \alpha$, an enhancement of the source function in the chromosphere (cf. Cram 1975) should be ruled out. Due to the dominance of the background radiation in the Balmer and Paschen continua contributing to the line source function, the latter is rather insensitive to temperature and electron density. Moustache profiles can be explained by a temperature increase and an opacity decrease, i.e. a partial evacuation, in the photosphere.

We propose that some of the bright features near and along dark mottles are produced by Cannon's (1970) channelling effect. The photons avoid the opacity of the clouds and escape preferentially from regions of low absorption. This finding could only be obtained from 2D non-LTE simulations.

Further studies will include the investigation of the $\mathrm{H} \alpha$ structures near the solar limb to better discriminate between elevated clouds and embedded features. Finally, time sequences of the $\mathrm{H} \alpha$ chromosphere, taken simultaneously with magnetograms, with the same high spatial resolution as used in the present study, will help to relate the dynamics of the chromospheric fine structures with the dynamics of the moustaches and the small-scale magnetic fields.

Acknowledgements. Part of this work was supported by the Deutsche Forschungsgemeinschaft through grant KN 152/26-1. J.H. acknowledges financial support from the Austrian Fonds zur Förderung der wissenschaftlichen Forschung (Erwin-SchrödingerRückkehrprogramm, R11). The Vacuum Tower Telescope is operated by the Kiepenheuer-Institut für Sonnenphysik, Freiburg, at the Spanish Observatorio del Teide of the Instituto de Astrofísica de Canarias. 


\section{References}

Al, N., Hirzberger, J., \& Kneer, F. 2003, Astron. Nachr./AN, 324, 364 Alissandrakis, C. E., Tsiropoula, G., \& Mein, P. 1990, A\&A, 230, 200 Athay, R. G. 1976, The Solar Chromosphere and Corona: Quiet Sun (Dordrecht: Reidel)

Auer, L., Fabiani Bendicho, P., \& Trujillo Bueno, J. 1994, A\&A, 292, 599

Beckers, J. M. 1964, Ph.D. Thesis, Utrecht

Beckers, J. M. 1972, ARA\&A, 10, 73

Bendlin, C., Volkmer, R., \& Kneer, F. 1992, A\&A, 257, 817

Bendlin, C., \& Volkmer, R. 1995, A\&AS, 112, 371

Bellot Rubio, L. R., Ruiz Cobo, B., \& Collados, M. 1997, ApJ, 478, L48

Brault, J. W., \& Neckel, H. 1987, Spectral Atlas of Solar Absolute Disk-averaged and Disk-center Intensities from 3290 to $12510 \AA$, unpublished

Cannon, C. J. 1970, ApJ, 161, 255

Christopoulou, E. B., Georgakilas, A. A., \& Koutchmy, S. 2001, Sol. Phys., 199, 61

Cram, L. E. 1975, Sol. Phys., 42, 53

Denker, C., de Boer, C. R., Volkmer, R., \& Kneer, F. 1995, A\&A, 296, 567

Dere, K. P., Bartoe, J.-D. F., \& Brueckner, G. E. 1989, Sol. Phys., 123, 41

Fabiani Bendicho, P., Kneer, F., \& Trujillo Bueno, J. 1992, A\&A, 264, 229

Fawzy, D., Rammacher, W., Ulmschneider, P., Musielak, Z. E., \& Stępień, K. 2002, A\&A, 386, 971

Grossmann-Doerth, U., \& von Uexküll, M. 1971, Sol. Phys., 20, 31

Grossmann-Doerth, U., \& von Uexküll, M. 1973, Sol. Phys., 28, 319

Heinzel, P., Mein, N., \& Mein, P. 1999, A\&A, 346, 322
Hirzberger, J., Koschinsky, M., Kneer, F., \& Ritter, C. 2001, A\&A, 367,1011

Innes, D. E., Inhester, B., Axford, W. I., \& Wilhelm, K. 1997, Nature, 386, 811

Koschinsky, M., Kneer, F., \& Hirzberger, J. 2001, A\&A, 365, 588

Lee, C. Y., Chae, J., \& Wang, H. 2000, ApJ, 545, 1124

Martres, M. J., \& Bruzek, A. 1977, in Illustrated Glossary for Solar and Solar Terrestrial Physics, ed. A. Bruzek, \& C. J. Durrant (Dordrecht: Reidel)

Matsuno, K., \& Hirayama, T. 1988, Sol. Phys., 117, 21

Mein, N., Mein, P., Heinzel, P., et al. 1996, A\&A, 309, 275

Mihalas, D. 1970, Stellar Atmospheres, first edtn. (San Francisco: Freeman)

Molowny-Horas, R., Heinzel, P., Mein, P., \& Mein, N. 1999, A\&A, 345,618

Ng, K. C. 1974, J. Chem. Phys., 61, 2680

Orrall, F. Q., \& Kopp, R. A. 1977, in The Energy Balance and Hydrodynamics of the Solar Chromosphere and Corona, ed. R.-M. Bonnet, \& P. Delache (Clermont-Ferrand: G. de Bussac), IAU Coll., 36, 457

Schmieder, B., Tziotziou, K., \& Heinzel, P. 2003, A\&A, 401, 361

Tsiropoula, G., Alissandrakis, C. E., \& Schmieder, B. 1993, A\&A, 271,574

Tsiropoula, G., Alissandrakis, C. E., \& Schmieder, B. 1994, A\&A, 290, 285

Vernazza, J. E., Avrett, E. H., \& Loeser, R. 1981, ApJS, 45, 635

von der Lühe, O. 1984, J. Opt. Soc. Am., A1, 510

von Uexküll, M., Kneer, F., Malherbe, J. M., \& Mein, P. 1989, A\&A, 208, 290

Weigelt, G. P. 1977, Optics Comm., 21, 55

Zachariadis, Th. G., Dara, H. C., Alissandrakis, C. E., Koutchmy, S., \& Contikakis, C. 2001, Sol. Phys., 202, 41 